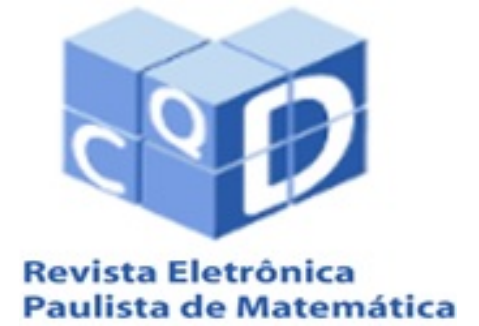

ISSN 2316-9664

Volume 8, dez. 2016

Fabricio Alves Oliveira

Instituto Federal de Educação, Ciência e Tecnologia de Minas Gerais

fabricio.oliveira@ifmg.edu.br

Dulce Mary de Almeida

Universidade Federal de

Uberlândia

dulce.almeida@ufu.br

\section{Superfícies regradas}

Ruled surfaces

\section{Resumo}

O objetivo deste trabalho é estudar uma importante classe de superfícies, constituída por aquelas que contém uma infinidade de retas: as Superfícies Regradas. Os exemplos mais óbvios de tais superfícies são dados pelos cones e cilindros. Além dessas, várias superfícies quádricas, incluindo o paraboloide hiperbólico e o hiperboloide de uma folha, são também superfícies regradas, embora este fato não seja tão natural. Estas superfícies são, indiscutivelmente, as mais fáceis de se parametrizar, bastando considerar apenas uma curva e um campo vetorial ao longo dessa curva. Além disso, veremos que em pontos regulares, a curvatura Gaussiana dessas superfícies é sempre não positiva. Em particular vamos analisar as superfícies regradas desenvolvíveis, que é uma subclasse especial das regradas. Mostraremos, que sua curvatura Gaussiana em pontos regulares é identicamente nula e apresentaremos um teorema de classificação parcial para estas superfícies.

\section{Palavras-chave:}

Superfície regrada. Curva de estricção. Superfície tangente. Curvatura gaussiana. Superfície regrada desenvolvível.

\begin{abstract}
The aim of this work is to study an important class of surfaces, constituted by those that contain an infinite number of straight lines. The most obvious examples of such surfaces are cones and cylinders. Besides these, several quadric surfaces, including the hyperbolic paraboloid and the hyperboloid of one leaf, are also ruled surfaces, although this fact is not so natural. These surfaces are undoubtedly the easiest to parameterize, considering only a curve and a vector field along this curve. In addition, we will see that at regular points, the Gaussian curvature of these surfaces is always non-positive. In particular we will analyze the developable ruled surfaces, which is a special subclass of the ruled surfaces. We will show that it's Gaussian curvature at regular points is identically null and we will present a partial classification theorem for these surfaces.
\end{abstract}

Keywords: Ruled surface. Striction curve. Tangent surface. Gaussian curvature. Developable ruled surface. 


\section{Introdução}

Superfícies diferenciáveis constituem uma ferramenta matemática de extrema valia, tanto do ponto de vista teórico quanto do ponto de vista prático, na resolução de diversos problemas que surgem de modo natural não só em geometria, mas em diversas áreas do conhecimento.

Em particular a classe das superfícies regradas, é um assunto clássico em Geometria Diferencial que, atualmente, tem despertado a atenção em diferentes áreas, como Geometria Diferencial Projetiva, Computação Gráfica e Desenho Industrial.

Neste trabalho, fazemos um estudo das Superfícies Regradas, superfícies com parametrizações extremamente simples e com várias propriedades interessantes. Os exemplos mais simples de superfícies regradas são os cilindros, os cones e as superfícies tangentes a uma curva. Também são exemplos de superfícies regradas o hiperboloide de uma folha, o paraboloide hiperbólico, o helicoide e a faixa de Möbius. Mostramos que, em pontos regulares, a curvatura Gaussiana de uma superfície regrada é sempre menor ou igual a zero, e é zero apenas ao longo das geratrizes que intersectam a linha de estricção em um ponto singular. No caso particular em que a superfície regrada é desenvolvível, a curvatura Gaussiana em pontos regulares é identicamente nula. Finalizamos o trabalho apresentando um teorema de classificação parcial das superfícies regradas desenvolvíveis.

\section{Definição e exemplos}

Uma superfície regrada é uma superfície gerada por uma reta movendo-se ao longo de uma curva $\alpha$. Mais precisamente, uma superfície regrada é a imagem (traço) de uma aplicação $X: I \times J \subseteq$ $\mathbb{R}^{2} \longrightarrow \mathbb{R}^{3}$, definida por

$$
X(t, v)=\alpha(t)+v w(t),
$$

em que $\alpha: I \longrightarrow \mathbb{R}^{3}$ e $w: I \longrightarrow \mathbb{R}^{3}-\{(0,0,0)\}$ são aplicações diferenciáveis. Nesse caso, dizemos que a superfície parametrizada por (1) é a superfície regrada gerada pela família a 1parâmetro de retas $\{\alpha(t), w(t)\}$.

As retas $L_{t}(v)=\alpha(t)+v w(t)$ e a curva $\alpha(t)$ são, respectivamente, as geratrizes e uma diretriz da superfície $X$. Os pontos $\left(t_{0}, v_{0}\right) \in I \times J$ tais que $X_{t}\left(t_{0}, v_{0}\right) \wedge X_{v}\left(t_{0}, v_{0}\right)=\overrightarrow{0}$, são chamados pontos singulares da superfície regrada.

Exemplo 1 (Cilindro Generalizado): Um cilindro generalizado é uma superfície regrada gerada por uma família a 1-parâmetro de retas $\{\alpha(t), w(t)\}, t \in I$, onde $\alpha(t)$ está contida em um plano $\pi$ e $w(t)$ é uma direção fixa em $\mathbb{R}^{3}$ não paralela à $\pi$. Assim, uma parametrização para o cilindro generalizado pode ser dada por

$$
X(t, v)=\alpha(t)+v q,
$$

onde $q \in \mathbb{R}^{3}-\{(0,0,0)\}$ é um vetor fixo não paralelo à $\pi$.

Exemplo 2 (Cone Generalizado): Um cone generalizado é uma superfície regrada gerada por uma família $\{\alpha(t), w(t)\}, t \in I$, em que $\alpha(t)$ está contida em um plano $\pi$ e todas as geratrizes $L_{t}$ passam por um ponto $p \in \mathbb{R}^{3}$ não pertencente ao plano $\pi$. O ponto $p$ é chamado vértice do cone. Como o vértice pertence a todas as geratrizes $L_{t}$, então, para cada $t \in I$, existe $v_{0}=v_{0}(t) \in \mathbb{R}$ tal que

$$
p=\alpha(t)+v_{0} w(t) \Longrightarrow \alpha(t)=p-v_{0} w(t) .
$$




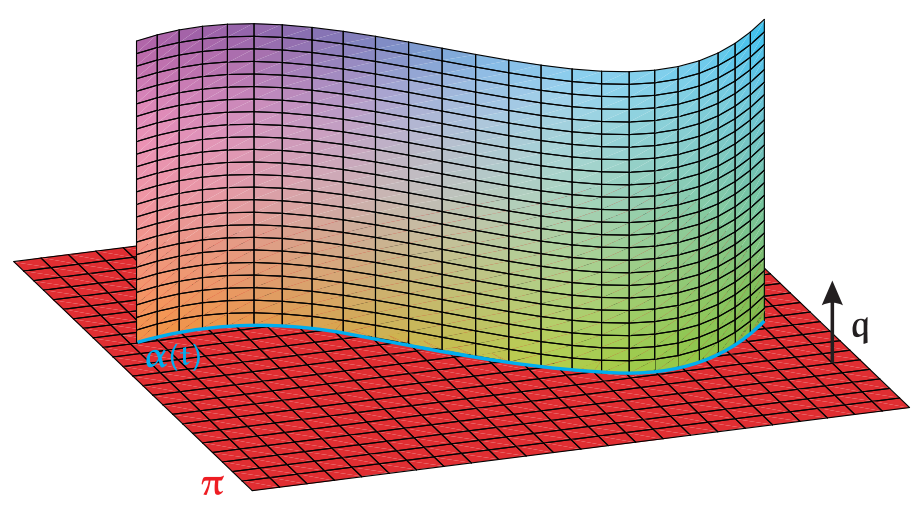

Figura 1: Cilindro Generalizado

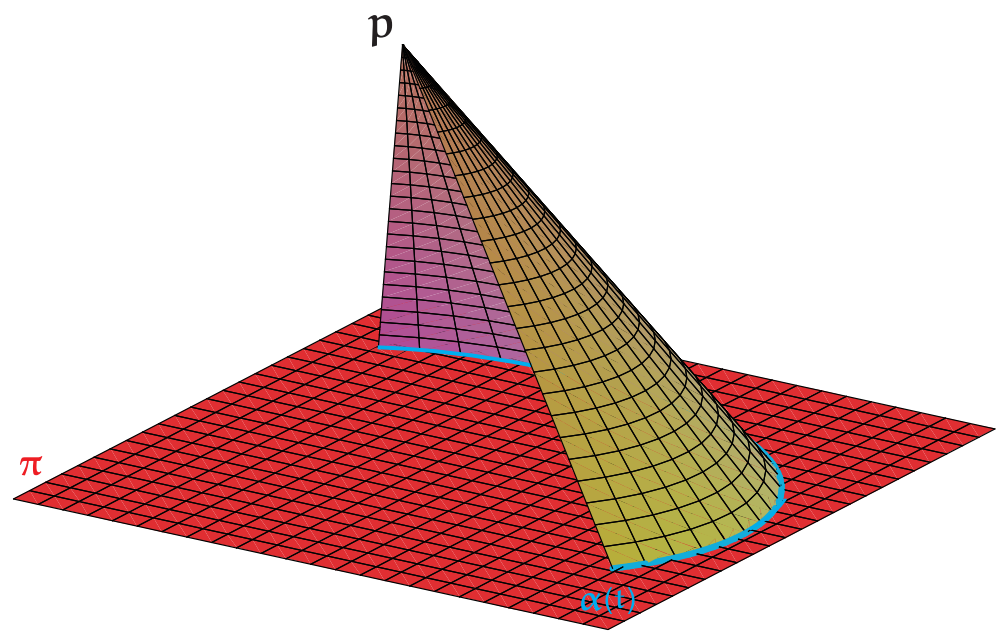

Figura 2: Cone Generalizado

Logo, uma parametrização para o cone generalizado é dada por

$$
X(t, v)=\alpha(t)+v w(t)=p-v_{0} w(t)+v w(t)=p+\left(v-v_{0}\right) w(t) .
$$

Fazendo $u(t, v)=v-v_{0}$, obtemos outra parametrização para o cone generalizado, dada por:

$$
X(t, u)=p+u w(t),
$$

onde $p$ é o vértice do cone.

Sob certas condições os cilindros e os cones generalizados são exemplos de superfícies regulares, como mostra as duas proposições a seguir:

Proposição 1 Seja $\alpha(u)=(x(u), y(u), z(u)), u \in I=(a, b)$, uma curva parametrizada regular contida no plano $\pi$. Se $w=(a, b, c)$ é um vetor não paralelo ao plano $\pi$, então

$$
X(u, v)=\alpha(u)+v w, \operatorname{com}(u, v) \in I \times \mathbb{R}=U
$$


é uma superfície parametrizada regular, cujo traço é um cilindro generalizado de diretriz $\alpha(I)$ e geratriz paralela à $w$. Se $\alpha$ é simples (isto é, se $\alpha$ é injetora), então $S=X(U)$ é uma superfície regular.

Demonstração: É claro que $X$ é diferenciável. Agora, a injetividade de $\alpha$ implica na injetividade de $X$. Assim, $X$ é um homeomorfismo sobre a sua imagem. Quanto à regularidade, temos que $X_{u}(u, v)=\alpha^{\prime}(u)$ é paralelo à $\pi$ e $X_{v}(u, v)=w$ que não é paralelo à $\pi$. Logo, $\left\{X_{u}, X_{v}\right\}$ é linearmente independente, ou seja, a condição de regularidade da definição de superfície regular está satisfeita. Portanto, $S=X(U)$ é uma superfície regular.

Proposição 2 Sejam $\alpha(u)=(x(u), y(u), z(u)), u \in I=(a, b)$, uma curva parametrizada regular plana e $p=(a, b, c)$ um ponto que não pertence ao plano que contém $\alpha$. Nos pontos em que $(1-v) \alpha^{\prime}(u) \times(p-\alpha(u))$ não se anula,

$$
X(u, v)=\alpha(u)+v(p-\alpha(u)),(u, v) \in I \times \mathbb{R}=U,
$$

é uma superfície parametrizada regular cujo traço é o cone generalizado $K$ de diretriz $\alpha(I)$ e vértice $p$. Quando $\alpha$ é uma curva simples, temos que $K-p$ é uma superfície regular (formada por duas componentes conexas).

Demonstração: Como $\alpha$ é simples, segue que $X$ é injetora e logo, é um homeomorfismo sobre sua imagem. Temos que $X_{u}(u, v)=\alpha^{\prime}(u)-v \alpha^{\prime}(u)=(1-v) \alpha^{\prime}(u)$ e $X_{v}(u, v)=p-\alpha(u)$. Assim, nos pontos em que $(1-v) \alpha^{\prime}(u) \times(p-\alpha(u))$ não se anula, a diferencial $d X_{q}$ é injetora. Portanto, nestas condições o traço de $X$ passa a ser uma superfície regular, que admite um atlas formado por $X$.

Exemplo 3 (Hiperboloide de uma folha): Sejam $\mathbb{S}^{1}$ o círculo unitário $x^{2}+y^{2}=1$ no plano $x y$, e $\alpha(s)$ uma parametrização de $\mathbb{S}^{1}$ pelo comprimento de arco. Para cada $s \in(-\varepsilon, 2 \pi+\varepsilon), \varepsilon>0$, considere $w(s)=\alpha^{\prime}(s)+e_{3}$, onde $e_{3}$ é o vetor unitário do eixo $z$. A superfície regrada gerada pela família $\{\alpha(s), w(s)\}$ é um hiperboloide de uma folha.

De fato, seja $\alpha(s)=(\cos (s), \operatorname{sen}(s), 0), s \in(0-\varepsilon, 2 \pi+\varepsilon), \varepsilon>0$ uma parametrização pelo comprimento de arco de $\mathbb{S}^{1}$. Assim, $\alpha^{\prime}(s)=(-\operatorname{sen}(s), \cos (s), 0)$ e

$$
\begin{aligned}
w(s) & =\alpha^{\prime}(s)+e_{3} \\
& =(-\operatorname{sen}(s), \cos (s), 0)+(0,0,1) \\
& =(-\operatorname{sen}(s), \cos (s), 1)
\end{aligned}
$$

Logo,

$$
\begin{aligned}
X(s, v) & =\alpha(s)+v w(s) \\
& =(\cos (s)-v \operatorname{sen}(s), \operatorname{sen}(s)+v \cos (s), v)
\end{aligned}
$$

Observe que $x(s, v)^{2}+y(s, v)^{2}-z(s, v)^{2}=1$, para todo $(s, v) \in(-\varepsilon, 2 \pi+\varepsilon) \times \mathbb{R}$. Isso mostra que o traço de $X(s, v)$ é um hiperboloide de uma folha, como queríamos.

Definição 3 Uma superfície regrada é dita duplamente regrada se possui duas parametrizações distintas. 
O hiperboloide de uma folha, do exemplo acima, é uma superfície duplamente regrada. De fato, se tomarmos $w(s)=-\alpha(s)+e_{3}$, teremos a superfície regrada

$$
X(s, v)=(\cos (s)+v \operatorname{sen}(s), \operatorname{sen}(s)-v \cos (s), v),
$$

cujo traço também satisfaz a equação $x^{2}+y^{2}-z^{2}=1$. Isso nos mostra que o hiperboloide de uma folha tem duas famílias de geratrizes.
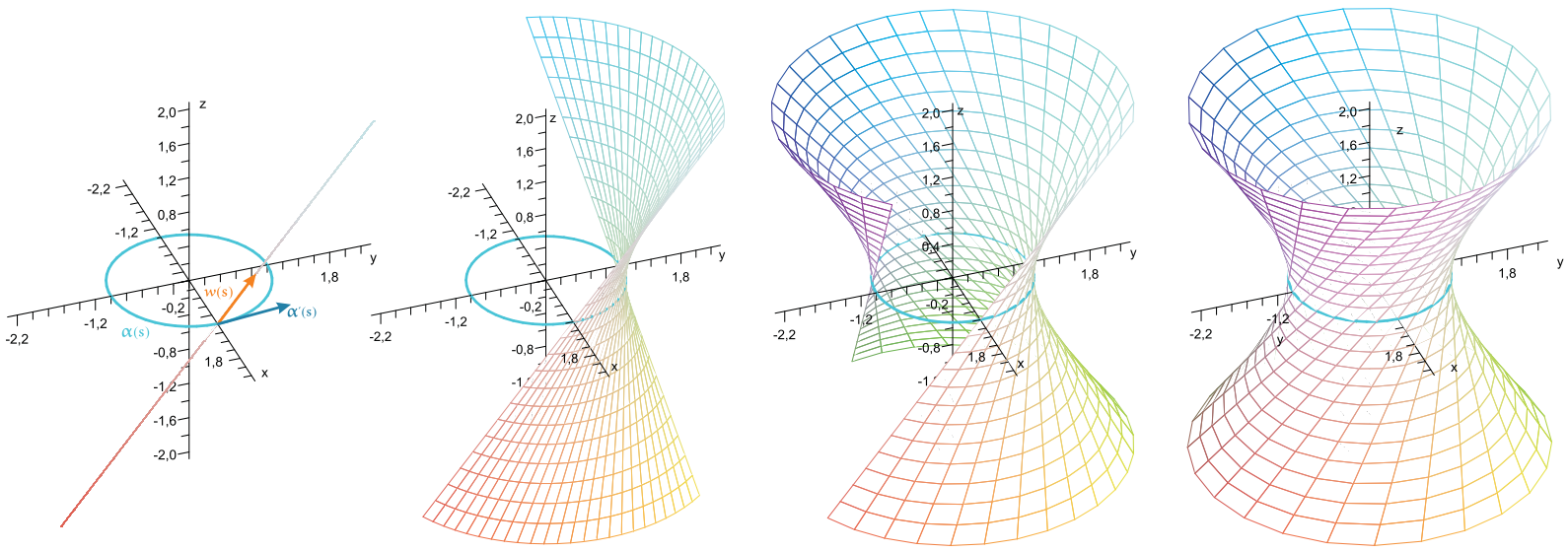

Figura 3: Hiperboloide de uma folha como superficie regrada
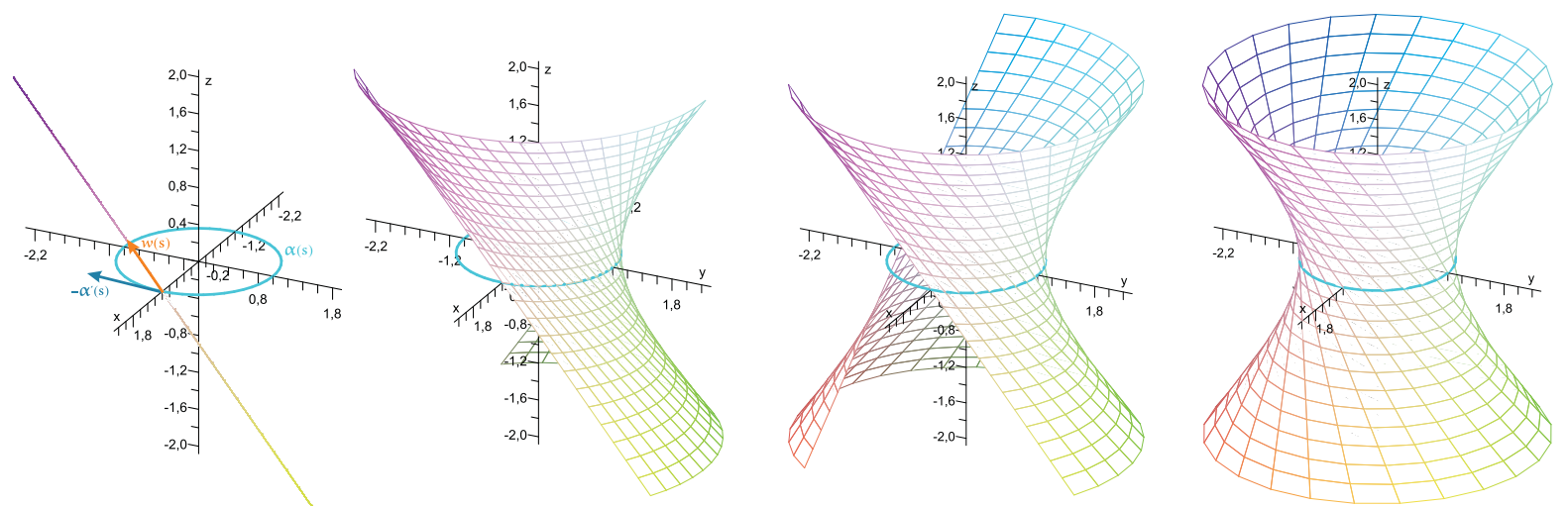

Figura 4: Outra família de geratrizes para o hiperboloide de uma folha

Exemplo 4 (Helicoide): O helicoide é uma superfície regrada gerada pela família $\{\alpha(t), w(t)\}$, em que $\alpha$ é a hélice parametrizada por $\alpha(t)=(\cos (t), \operatorname{sen}(t), t)$ e $w(t)=(\cos (t), \operatorname{sen}(t), 0), t \in \mathbb{R}$, ou seja, é a superfície formada por todas as retas paralelas ao plano $x y$, que unem cada ponto do eixo $z$ com o ponto da hélice situado à mesma altura. Assim, uma parametrização para o helicoide é dada por: 


$$
\begin{aligned}
X(t, v) & =\alpha(t)+v w(t) \\
& =(\cos (t), \operatorname{sen}(t), t)+v(\cos (t), \operatorname{sen}(t), 0) \\
& =((1+v) \cos (t),(1+v) \operatorname{sen}(t), t),(t, v) \in \mathbb{R}^{2} .
\end{aligned}
$$
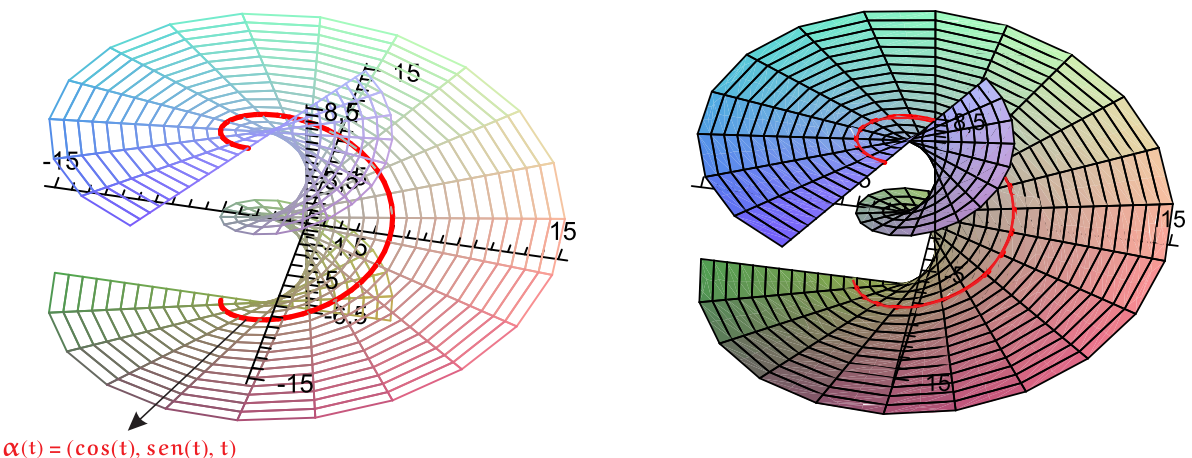

Figura 5: Helicoide

Exemplo 5 (Superfícies Tangentes): Seja $\alpha: I \longrightarrow \mathbb{R}^{3}$ uma curva parametrizada regular. A superfície

$$
X(t, v)=\alpha(t)+v \alpha^{\prime}(t),(t, v) \in I \times \mathbb{R},
$$

é a superfície regrada gerada pela família a 1-parâmetro de retas $\left\{\alpha(t), \alpha^{\prime}(t)\right\}$, denominada superfície tangente da curva $\alpha$.

Vamos obter a superfície tangente da Curva de Viviani, curva obtida pela intersecção do cilindro $(x-a)^{2}+y^{2}=a^{2}$ com a esfera $x^{2}+y^{2}+z^{2}=(2 a)^{2}$, em que $a$ é um número real positivo.
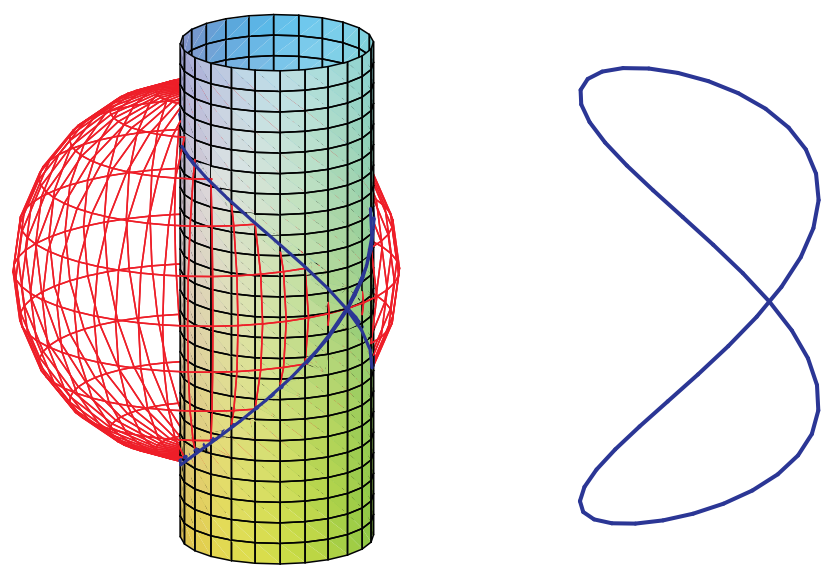

Figura 6: Curva de Viviani

Temos que uma parametrização para este cilindro é dada por

$$
Y(t, z)=(x(t), y(t), z)=(a(1+\cos (t)), a \operatorname{sen}(t), z),
$$


com $t \in(0-\varepsilon, 2 \pi+\varepsilon), \varepsilon>0$ e $z \in \mathbb{R}$. Substituindo expressão na equação da esfera $x^{2}+y^{2}+$ $z^{2}=4 a^{2}$, e utilizando a identidade $\cos (t)=1-2 \operatorname{sen}^{2}\left(\frac{t}{2}\right)$, obtemos $z=z(t)=2 a \operatorname{sen}\left(\frac{t}{2}\right), t \in$ $(0-\varepsilon, 4 \pi+\varepsilon), \varepsilon>0$. Assim,

$$
\beta(t)=\left(a(1+\cos (t)), a \operatorname{sen}(t), 2 a \operatorname{sen}\left(\frac{t}{2}\right)\right), t \in(0-\varepsilon, 4 \pi+\varepsilon), \varepsilon>0,
$$

é uma parametrização para a Curva de Viviani. Veja que:

$$
\beta^{\prime}(t)=\left(-a \operatorname{sen}(t), a \cos (t), a \cos \left(\frac{t}{2}\right)\right) \neq(0,0,0), \forall t \in(0-\varepsilon, 4 \pi+\varepsilon), \varepsilon>0,
$$

ou seja, $\beta(t)$ é regular. Portanto, a superfície tangente à Curva de Viviani é dada por:

$$
\begin{aligned}
X(t, v) & =\beta(t)+v \beta^{\prime}(t) \\
& =a\left(1+\cos (t)-v \operatorname{sen}(t), \operatorname{sen}(t)+v \cos (t), 2 \operatorname{sen}\left(\frac{t}{2}\right)+v \cos \left(\frac{t}{2}\right)\right),
\end{aligned}
$$

$\operatorname{com}(t, v) \in(0-\varepsilon, 4 \pi+\varepsilon) \times \mathbb{R}, \varepsilon>0$.
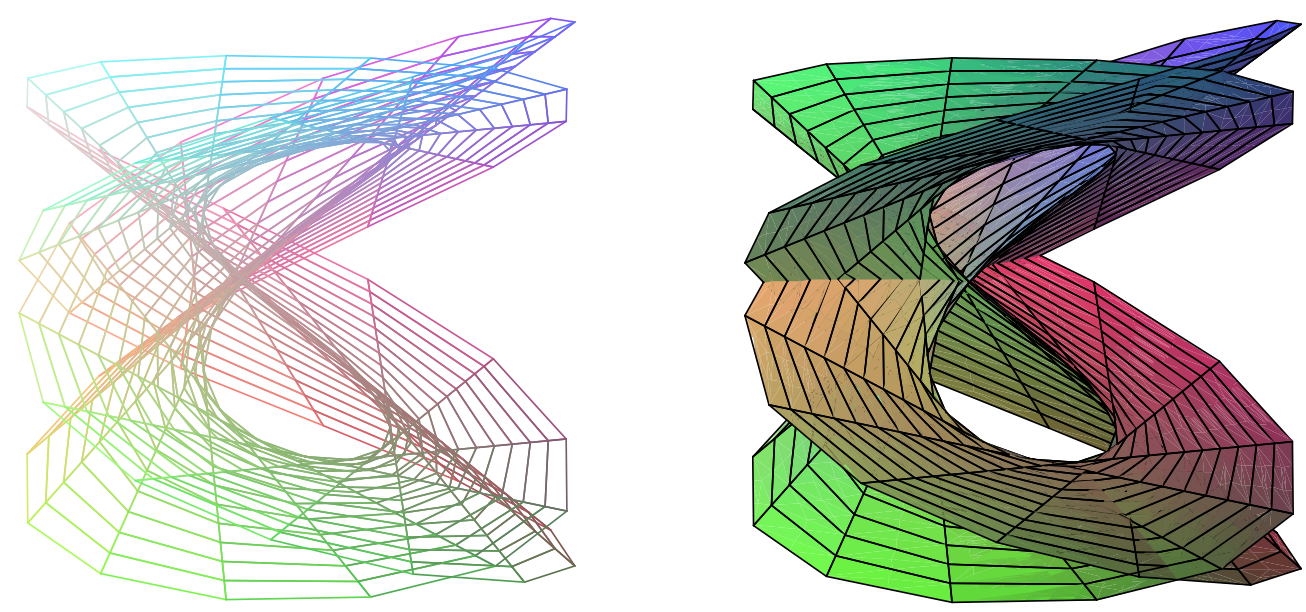

Figura 7: Superfície Tangente à Curva de Viviani

Observação: Definimos superfícies regradas de modo a permitir a ocorrência de singularidades. Isso é necessário se queremos incluir superfícies tangentes (que podem ter auto-intersecções) e cones.

Exemplo 6 (Guarda-chuva de Whitney): O guarda-chuva de Whitney é a imagem da aplicação $f: I \times J \longrightarrow \mathbb{R}^{3}$, definida por $f(x, y)=\left(x^{2}, y, x y\right)$, onde $I$ e $J$ são intervalos contendo a origem.

Vamos mostrar que o guarda-chuva de Whitney é uma superfície regrada. Para isso, considere as aplicações diferenciáveis $\alpha(t)=\left(t^{2}, 0,0\right)$ e $w(t)=\left(0, \frac{1}{\sqrt{1+t^{2}}}, \frac{t}{\sqrt{1+t^{2}}}\right), t \in I$. Definindo a superfície regrada gerada pela família a 1-parâmetro de retas $\{\alpha(t), w(t)\}$, obtemos: 


$$
\begin{aligned}
X(t, v) & =\alpha(t)+v w(t) \\
& =\left(t^{2}, 0,0\right)+v\left(0, \frac{1}{\sqrt{1+t^{2}}}, \frac{t}{\sqrt{1+t^{2}}}\right) \\
& =\left(t^{2}, \frac{v}{\sqrt{1+t^{2}}}, \frac{t v}{\sqrt{1+t^{2}}}\right),(t, v) \in I \times J .
\end{aligned}
$$

Agora, considerando a mudança de variáveis

$$
\left\{\begin{array}{l}
x(t, v)=t \\
y(t, v)=\frac{v}{\sqrt{1+t^{2}}}
\end{array},\right.
$$

temos que $f(x(t, v), y(t, v))=X(t, v)$, ou seja, o guarda-chuva de Whitney é uma superfície regrada.
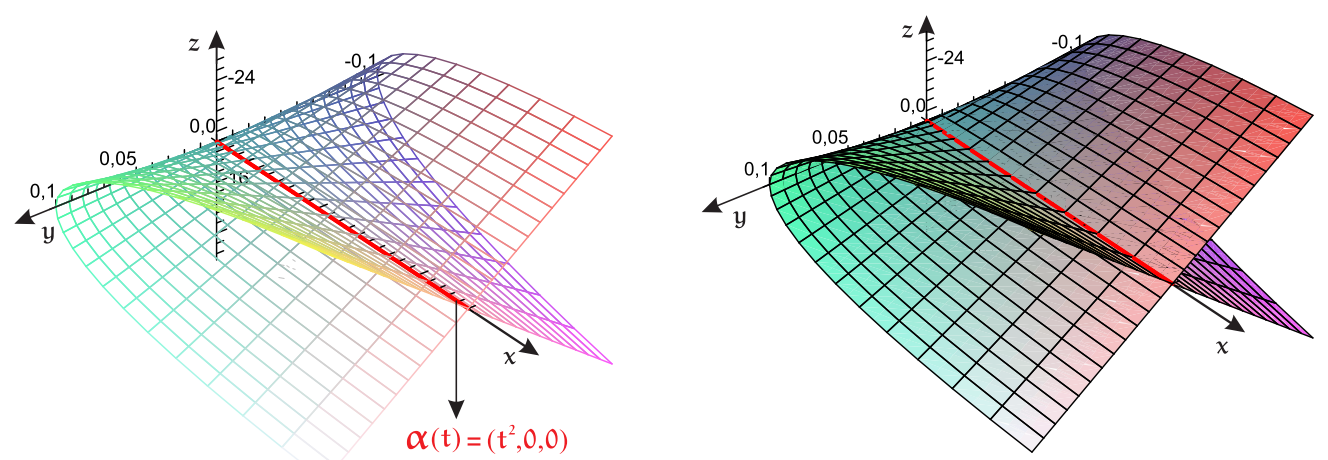

Figura 8: Guarda-chuva de Whitney

Exemplo 7 (Paraboloide Hiperbólico): Vamos mostrar que o paraboloide hiperbólico

$$
S=\left\{(x, y, z) \in \mathbb{R}^{3} ; \frac{x^{2}}{a^{2}}-\frac{y^{2}}{b^{2}}=z, \operatorname{com} a, b \in \mathbb{R}^{*}\right\}
$$

é uma superfície regrada. Inicialmente, observe que, fazendo uma mudança de variáveis adequada, podemos representar o paraboloide $S$ num sistema de coordenadas ortogonais $(u, v, w)$ de modo que sua equação seja dada por $w=k u v, k \neq 0$. Mais precisamente, trocando o sistema de coordenadas $(x, y, z)$ por $(b x, a y, z)$ e fazendo uma rotação desse novo sistema de coordenadas de um ângulo de $\frac{\pi}{4}$ radianos, em torno do eixo $z$, no sentido horário, obtemos:

$$
\left(\begin{array}{c}
u \\
v \\
w
\end{array}\right)=\left(\begin{array}{ccc}
\cos \left(\frac{\pi}{4}\right) & \operatorname{sen}\left(\frac{\pi}{4}\right) & 0 \\
-\operatorname{sen}\left(\frac{\pi}{4}\right) & \cos \left(\frac{\pi}{4}\right) & 0 \\
0 & 0 & 1
\end{array}\right)\left(\begin{array}{c}
b x \\
a y \\
z
\end{array}\right) \Longrightarrow\left\{\begin{array}{l}
u=\frac{\sqrt{2}}{2} b x+\frac{\sqrt{2}}{2} a y \\
v=-\frac{\sqrt{2}}{2} b x+\frac{\sqrt{2}}{2} a y \\
w=z
\end{array}\right.
$$


Logo, $x=\frac{u-v}{b \sqrt{2}}, y=\frac{u+v}{a \sqrt{2}}$ e $z=w$. Assim, a equação do paraboloide no novo sistema de coordenadas ortogonais é dada por

$$
w=\underbrace{\frac{-2}{a^{2} b^{2}}}_{=k} u v \Longrightarrow w=k u v, k \neq 0 .
$$

Portanto, todo paraboloide hiperbólico $z=\frac{x^{2}}{a^{2}}-\frac{y^{2}}{b^{2}}$, em que $a, b \in \mathbb{R}^{*}$, pode ser representado em um sistema de coordenadas ortogonais $(\bar{x}, \bar{y}, \bar{z})$ de modo que sua equação seja dada por $\bar{z}=$ $k \bar{x} \bar{y}, k \neq 0$.

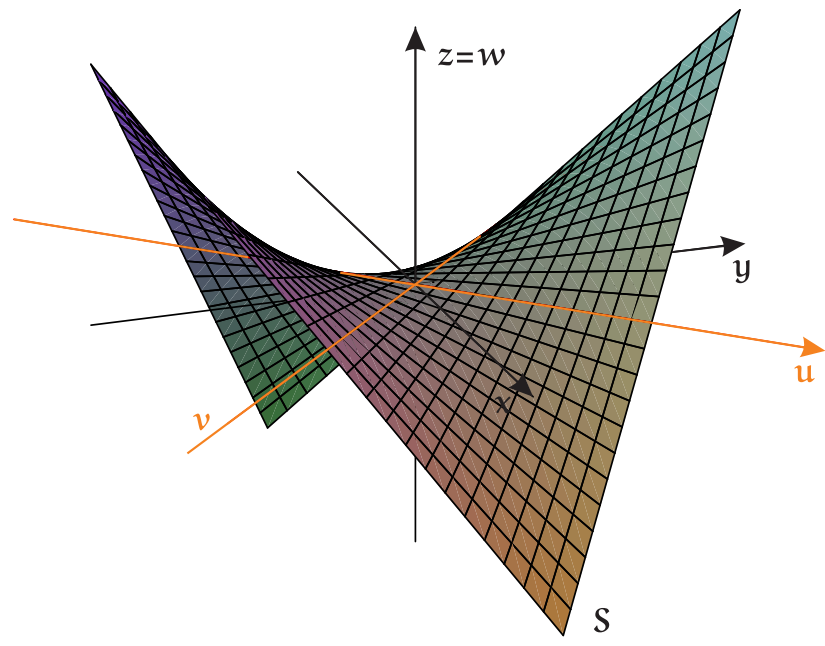

Figura 9: Representação do Paraboloide Hiperbólico $w=k u v, k \neq 0$

Vamos exibir uma família a 1-parâmetro de retas $\{\alpha(t), w(t)\}$ para o paraboloide hiperbólico $z=k x y, k \neq 0$. Para isso, observe que as retas $y=\frac{z}{t k}, x=t$, para cada $t \neq 0$ pertencem ao paraboloide em questão. Considerando a intersecção desta família de retas com o plano $z=0$, obtemos a curva $\alpha(t)=(t, 0,0)$. Tomando esta curva como diretriz e os vetores unitários $w(t)$ paralelos às retas $y=\frac{z}{t k}, x=t$, obtemos a família a 1-parâmetro de retas $\{\alpha(t), w(t)\}$, sendo $\alpha(t)=(t, 0,0)$ e $w(t)=\frac{(0,1, k t)}{\sqrt{1+k^{2} t^{2}}}$, que gera a superfície regrada

$$
\begin{aligned}
X(t, v) & =\alpha(t)+v w(t) \\
& =\left(t, \frac{v}{\sqrt{1+k^{2} t^{2}}}, \frac{v k t}{\sqrt{1+k^{2} t^{2}}}\right), t, v \in \mathbb{R}
\end{aligned}
$$

cujo traço coincide com $S$.

Vamos fazer agora, o estudo de algumas propriedades das superfícies regradas. Para isso vamos precisar da seguinte definição:

Definição 4 Uma superfície regrada parametrizada por $X(t, v)=\alpha(t)+v w(t),(t, v) \in I \times J \subset \mathbb{R}^{2}$, é dita não-cilíndrica, se $w^{\prime}(t) \neq \overrightarrow{0}$, para todo $t \in I$. 

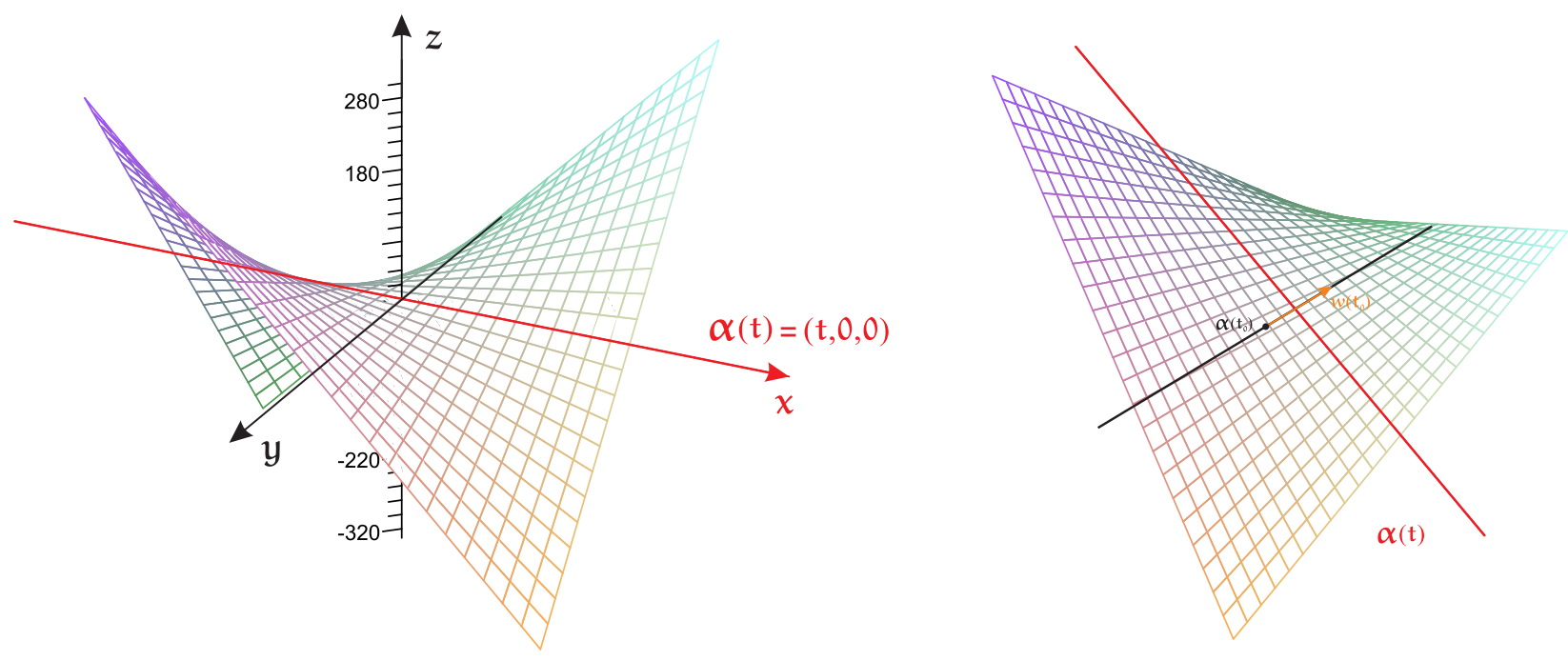

Figura 10: Paraboloide Hiperbólico Regrado (vistas lateral e superior)

\section{Curva de Estricção e suas Propriedades}

Lema 5 Seja $\widetilde{X}: I \times J \longrightarrow \mathbb{R}^{3}$, definida por $\widetilde{X}(t, v)=\alpha(t)+v \gamma(t)$, uma parametrização de uma superfície regrada não-cilíndrica. Então, $\widetilde{X}$ possui uma reparametrização da forma

$$
X(t, v)=\beta(t)+v w(t),
$$

onde $\|w(t)\| \equiv 1$ e $\left\langle\beta^{\prime}(t), w^{\prime}(t)\right\rangle=0$.

Demonstração: Por definição, a função diferenciável $\gamma$ nunca se anula. Logo, podemos definir uma parametrização $\widetilde{\widetilde{X}}$ de $\widetilde{X}$ dada por

$$
\widetilde{\widetilde{X}}(t, v)=\widetilde{X}\left(t, \frac{v}{\|\gamma(t)\|}\right)=\alpha(t)+v \frac{\gamma(t)}{\|\gamma(t)\|} .
$$

Claramente, $\widetilde{\widetilde{X}}$ tem o mesmo traço de $\widetilde{X}$. Fazendo, $w(t)=\frac{\gamma(t)}{\|\gamma(t)\|}$, podemos escrever,

$$
\widetilde{\widetilde{X}}(t, v)=\alpha(t)+v w(t) .
$$

Como $\|w(t)\|=1$, segue que $\left\langle w(t), w^{\prime}(t)\right\rangle=0$. Queremos obter uma curva parametrizada $\beta$, contida no traço da superfície regrada, de modo que $\left\langle\beta^{\prime}(t), w^{\prime}(t)\right\rangle=0$, para todo $t \in I$. Assim, devemos ter

$$
\beta(t)=\alpha(t)+\mu(t) w(t)
$$

para alguma função a valores reais $\mu=\mu(t)$.

Supondo a existência de uma tal curva $\beta$, temos que:

$$
\beta^{\prime}(t)=\alpha^{\prime}(t)+\mu^{\prime}(t) w(t)+\mu(t) w^{\prime}(t)
$$

Daí, 


$$
0=\left\langle\beta^{\prime}(t), w^{\prime}(t)\right\rangle=\left\langle\alpha^{\prime}(t), w^{\prime}(t)\right\rangle+\mu^{\prime}(t) \underbrace{\left\langle w(t), w^{\prime}(t)\right\rangle}_{=0}+\mu(t)\left\langle w^{\prime}(t), w^{\prime}(t)\right\rangle .
$$

Como a superfície regrada é não-cilíndrica, $\gamma^{\prime}(t) \neq \overrightarrow{0}$, para todo $t \in I$ e, consequentemente, $w^{\prime}(t)$ nunca se anula. Logo, podemos definir $\mu=\mu(t)$ por

$$
\mu(t)=-\frac{\left\langle\alpha^{\prime}(t), w^{\prime}(t)\right\rangle}{\left\langle w^{\prime}(t), w^{\prime}(t)\right\rangle}=-\frac{\left\langle\alpha^{\prime}(t), w^{\prime}(t)\right\rangle}{\left\|w^{\prime}(t)\right\|^{2}} .
$$

Assim, se definirmos $\beta: I \subset J \longrightarrow \widetilde{\widetilde{X}}$, por

$$
\beta(t)=\alpha(t)-\frac{\left\langle\alpha^{\prime}(t), w^{\prime}(t)\right\rangle}{\left\|w^{\prime}(t)\right\|^{2}} w(t),
$$

obtemos uma curva parametrizada, contida na superfície, satisfazendo $\left\langle\beta^{\prime}(t), w(t)\right\rangle=0$.

Por fim, definindo

$$
\begin{aligned}
X(t, v) & =\widetilde{\widetilde{X}}(t, \mu(t)+v) \\
& =\alpha(t)+(\mu(t)+v) w(t) \\
& =\underbrace{\alpha(t)+\mu(t) w(t)}_{\beta(t)}+v w(t)=\beta(t)+v w(t),
\end{aligned}
$$

obtemos uma reparametrização de $\widetilde{\widetilde{X}}$, consequentemente, uma reparametrização de $\widetilde{X}$, satisfazendo as condições desejadas.

Definição 6 Seja $X$ uma superfície regrada não-cilíndrica dada por $X(t, v)=\alpha(t)+v w(t)$. A curva

$$
\beta(t)=\alpha(t)-\frac{\left\langle\alpha^{\prime}(t), w^{\prime}(t)\right\rangle}{\left\|w^{\prime}(t)\right\|^{2}} w(t)
$$

é chamada curva de estricção de $X$, e seus pontos são chamados de pontos centrais da superfície regrada.

Proposição 7 A curva de estricção $\beta(t)$ de uma superfície regrada não-cilíndrica $X$, não depende da escolha da diretriz $\alpha$ da superfície regrada.

Demonstração: Seja $\widetilde{\alpha}$ uma outra diretriz da superfície regrada $X$, ou seja, para todo $(t, v) \in I \times J$,

$$
X(t, v)=\alpha(t)+v w(t)=\widetilde{\alpha}+s w(t)
$$

para alguma função $s=s(v)$. Assim, temos que

$$
\beta(t)=\alpha(t)-\frac{\left\langle\alpha^{\prime}(t), w^{\prime}(t)\right\rangle}{\left\|w^{\prime}(t)\right\|^{2}} w(t)
$$

e 


$$
\widetilde{\beta}(t)=\widetilde{\alpha}(t)-\frac{\left\langle\widetilde{\alpha}^{\prime}(t), w^{\prime}(t)\right\rangle}{\left\|w^{\prime}(t)\right\|^{2}} w(t),
$$

são as curvas de estricção correspondentes à $\alpha(t)$ e $\widetilde{\alpha}(t)$, respectivamente. Logo,

$$
\beta(t)-\widetilde{\beta}(t)=(\alpha(t)-\widetilde{\alpha}(t))+\frac{\left\langle\widetilde{\alpha}^{\prime}(t)-\alpha^{\prime}(t), w^{\prime}(t)\right\rangle}{\left\|w^{\prime}(t)\right\|^{2}} w(t) .
$$

Por outro lado, de (2), temos que:

$$
\alpha(t)-\widetilde{\alpha}(t)=(s-v) w(t)
$$

Substituindo essa expressão em (3), obtemos

$$
\beta(t)-\widetilde{\beta}(t)=\left[(s-v)+\frac{\left\langle(v-s) w^{\prime}(t), w^{\prime}(t)\right\rangle}{\left\langle w^{\prime}(t), w^{\prime}(t)\right\rangle}\right] w(t)=\overrightarrow{0}
$$

ou seja, $\beta(t)=\widetilde{\beta}(t)$, para todo $t$ em $I$.

Do exposto acima, concluímos que sempre podemos tomar a curva de estricção como a diretriz de uma superfície regrada não-cilíndrica.

\subsection{Exemplos de curvas de estricção}

(1) Vamos obter a curva de estricção $\beta(t)$ do cone generalizado parametrizado por $X(t, v)=$ $p+v w(t)$. Temos que

$$
\alpha(t)=p \Longrightarrow \alpha^{\prime}(t)=\overrightarrow{0}
$$

Daí,

$$
\mu(t)=-\frac{\left\langle\alpha^{\prime}(t), w^{\prime}(t)\right\rangle}{\left\|w^{\prime}(t)\right\|^{2}}=\frac{\left\langle\overrightarrow{0}, w^{\prime}(t)\right\rangle}{\left\|w^{\prime}(t)\right\|^{2}}=0
$$

Logo,

$$
\begin{aligned}
\beta(t) & =\alpha(t)+\mu(t) w(t) \\
& =p+0 w(t)=p
\end{aligned}
$$

para todo $t \in I$, ou seja, a curva de estricção do cone generalizado é degenerada e é o seu vértice.

(2) Consideremos o hiperboloide de uma folha

$$
X(s, v)=(\cos (s)-v \operatorname{sen}(s), \operatorname{sen}(s)+v \cos (s), v)
$$

gerado por $\alpha(s)=(\cos (s), \operatorname{sen}(s), 0)$ e $w(s)=(-\operatorname{sen}(s), \cos (s), 1), s \in(0-\varepsilon, 2 \pi+\varepsilon), \varepsilon>0$. É claro que $\alpha(s)$ é uma curva contida em $X$ e

$$
\left\langle\alpha^{\prime}(s), w^{\prime}(s)\right\rangle=\langle(-\operatorname{sen}(s), \cos (s), 0),(-\cos (s),-\operatorname{sen}(s), 0)\rangle=0, \forall s \in(0-\varepsilon, 2 \pi+\varepsilon), \varepsilon>0,
$$

isto é, a curva de estricção $\beta$ do hiperboloide de uma folha é a própria diretriz $\alpha$, ou seja, é a circunferência $x^{2}+y^{2}=1$ no plano $z=0$. 
(3) Seja $\alpha: I \longrightarrow \mathbb{R}^{3}$ uma curva parametrizada regular. Consideremos a superfície tangente à curva $\alpha$, dada por

$$
X(s, v)=\alpha(s)+v \alpha^{\prime}(s),(s, v) \in I \times \mathbb{R} .
$$

Vamos mostrar que a curva de estricção $\beta$ das superfícies tangentes coincide com a curva da qual se está construindo a superfície tangente, isto é, vamos mostrar que $\beta(s)=\alpha(s)$, para todo $s$ em I. De fato, sem perda de generalidade, suponha $\alpha$ parametrizada pelo comprimento de arco, ou seja, $\left\|\alpha^{\prime}(s)\right\|=1, \forall s \in I$. Temos que

$$
w(s)=\alpha^{\prime}(s) \Longrightarrow w^{\prime}(s)=\alpha^{\prime \prime}(s)
$$

Como $\alpha^{\prime}(s)$ é unitário, temos que $\left\langle\alpha^{\prime}(s), \alpha^{\prime \prime}(s)\right\rangle=0$, para todo $s$. Logo,

$$
\mu(s)=-\frac{\left\langle\alpha^{\prime}(s), w^{\prime}(s)\right\rangle}{\left\|w^{\prime}(s)\right\|^{2}}=-\frac{\left\langle\alpha^{\prime}(s), \alpha^{\prime \prime}(s)\right\rangle}{\left\|\alpha^{\prime \prime}(s)\right\|^{2}}=0 .
$$

Portanto,

$$
\beta(s)=\alpha(s)+\mu(s) w(s)=\alpha(s)+0 w(s)=\alpha(s),
$$

para todo $s$ em $I$, como queríamos.

Assim, no Exemplo 5, a curva de estricção da Superfície Tangente à Curva de Viviani é dada pela própria Curva de Viviani.

(4) Agora, vamos obter a curva de estricção do helicoide

$$
X(t, v)=((1+v) \cos (t),(1+v) \operatorname{sen}(t), t),(t, v) \in \mathbb{R}^{2} .
$$

Vimos que ele é gerado pelas curvas $\alpha(t)=(\cos (t), \operatorname{sen}(t), t)$ e $w(t)=(\cos (t), \operatorname{sen}(t), 0)$. Então, temos que $\alpha^{\prime}(t)=(-\operatorname{sen} t(t), \cos (t), 1)$ e $w^{\prime}(t)=(-\operatorname{sen}(t), \cos (t), 0)$. Logo,

$$
\begin{aligned}
\mu(t) & =-\frac{\left\langle\alpha^{\prime}(t), w^{\prime}(t)\right\rangle}{\left\|w^{\prime}(t)\right\|^{2}} \\
& =-\frac{\langle(-\operatorname{sent}(t), \cos (t), 1),(-\operatorname{sen}(t), \cos (t), 0)\rangle}{1} \\
& =-\left(\operatorname{sen}^{2}(t)+\cos ^{2}(t)\right)=-1 .
\end{aligned}
$$

Assim, a curva de estricção do helicoide é dada por

$$
\beta(t)=\alpha(t)+\mu(t) w(t)=\alpha(t)-w(t)=(0,0, t), t \in \mathbb{R}
$$

isto é, a curva de estricção do helicoide acima é o eixo $z$.

Portanto, uma parametrização para o helicoide usando a sua curva de estricção como diretriz é dada por

$$
\begin{aligned}
Y(t, v) & =(0,0, t)+v(\cos (t), \operatorname{sen}(t), 0) \\
& =(v \cos (t), v \operatorname{sen}(t), t), \quad(t, v) \in \mathbb{R}^{2}
\end{aligned}
$$




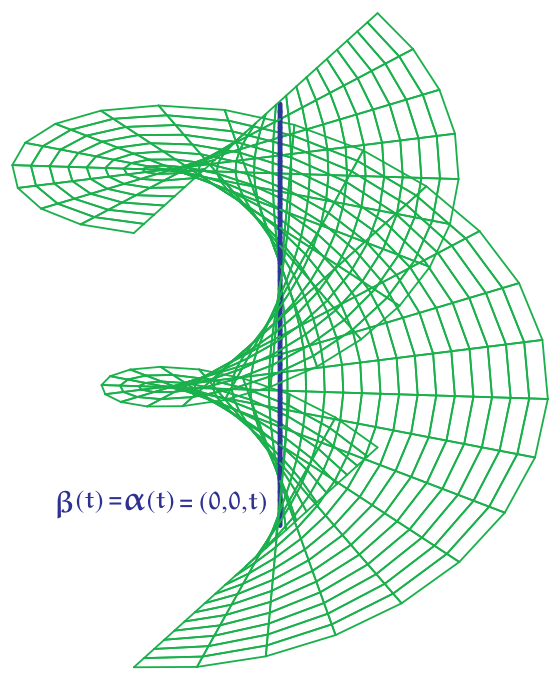

Figura 11: Curva de Estricção do Helicoide

(5) Vimos que o guarda-chuva de Whitney é uma superfície regrada gerada por $\alpha(t)=\left(t^{2}, 0,0\right)$ $\mathrm{e} w(t)=\left(0, \frac{1}{\sqrt{1+t^{2}}}, \frac{t}{\sqrt{1+t^{2}}}\right)$. Assim,

$$
\begin{aligned}
& \alpha^{\prime}(t)=(2 t, 0,0) \\
& w^{\prime}(t)=\left(0, \frac{-t}{\left(1+t^{2}\right)^{\frac{3}{2}}}, \frac{1}{\left(1+t^{2}\right)^{\frac{3}{2}}}\right) .
\end{aligned}
$$

Logo,

$$
\mu(t)=-\frac{\left\langle\alpha^{\prime}(t), w^{\prime}(t)\right\rangle}{\left\|w^{\prime}(t)\right\|^{2}}=0,
$$

e, assim, a curva de estricção do guarda-chuva de Whitney é a curva diretriz da nossa parametrização,

$$
\beta(t)=\alpha(t)=\left(t^{2}, 0,0\right), t \in I .
$$

(6) Por fim, veja que a curva de estricção do paraboloide hiperbólico do Exemplo 7 é o eixo $x$. De fato, vimos que o paraboloide hiperbólico é uma superfície regrada gerada pela família

$$
\alpha(t)=(t, 0,0) \text { e } w(t)=\frac{(0,1, k t)}{\sqrt{1+k^{2} t^{2}}} .
$$

A curva $\alpha$ está contida no paraboloide e satisfaz $\left\langle\alpha^{\prime}(t), w^{\prime}(t)\right\rangle=0$, para todo $t$. Assim, por definição, a diretriz $\alpha(t)=(t, 0,0)$ é a curva de estricção do paraboloide hiperbólico.

Teorema 8 Em pontos regulares, a curvatura Gaussiana $K$ de uma superfície regrada não-cilíndrica satisfaz $K \leq 0$, e é zero apenas ao longo das geratrizes que intersectam a curva de estricção em um ponto singular. 
Demonstração: Sem perda de generalidade, podemos supor a superfície regrada não-cilíndrica parametrizada por

$$
X(t, u)=\beta(t)+u w(t), \quad(t, u) \in I \times J,
$$

sendo $\beta(t)$ a sua curva de estricção, $w^{\prime}(t) \neq \overrightarrow{0}$ e $\|w(t)\|=1$, para todo $t \in I$. Derivando essa expressão, com respeito a $t$ e a $u$, obtemos:

$$
X_{t}(t, u)=\beta^{\prime}(t)+u w^{\prime}(t) \text { e } X_{u}(t, u)=w(t)
$$

Daí,

$$
X_{t}(t, u) \wedge X_{u}(t, u)=\beta^{\prime}(t) \wedge w(t)+u w^{\prime}(t) \wedge w(t) .
$$

Como $\left\langle w^{\prime}(t), w(t)\right\rangle=0$ e $\left\langle w^{\prime}(t), \beta^{\prime}(t)\right\rangle=0$, concluímos que

$$
\beta^{\prime}(t) \wedge w(t)=\lambda(t) w^{\prime}(t)
$$

para alguma função $\lambda(t)$. Assim, $X_{t}(t, u) \wedge X_{u}(t, u)=\lambda(t) w^{\prime}(t)+u w^{\prime}(t) \wedge w(t)$ e então,

$$
\begin{aligned}
\left\|X_{t}(t, u) \wedge X_{u}(t, u)\right\|^{2}= & \left\|\lambda(t) w^{\prime}(t)+u w^{\prime}(t) \wedge w(t)\right\|^{2} \\
= & \left\langle\lambda(t) w^{\prime}(t)+u w^{\prime}(t) \wedge w(t), \lambda(t) w^{\prime}(t)+u w^{\prime}(t) \wedge w(t)\right\rangle \\
= & \left\langle\lambda(t) w^{\prime}(t), \lambda w^{\prime}(t)\right\rangle+\left\langle\lambda(t) w^{\prime}(t), u w^{\prime}(t) \wedge w(t)\right\rangle \\
& +\left\langle u w^{\prime}(t) \wedge w(t), \lambda w^{\prime}(t)\right\rangle+\left\langle u w^{\prime}(t) \wedge w(t), u w^{\prime}(t) \wedge w(t)\right\rangle \\
= & \lambda^{2}(t) \underbrace{\left\langle w^{\prime}(t), w^{\prime}(t)\right\rangle}_{\left\|w^{\prime}(t)\right\|^{2}}+2 \lambda(t) u \underbrace{\left\langle w^{\prime}(t), w^{\prime}(t) \wedge w(t)\right\rangle}_{=0} \\
& +u^{2}\left\langle w^{\prime}(t) \wedge w(t), w^{\prime}(t) \wedge w(t)\right\rangle .
\end{aligned}
$$

Veja que:

$$
\left\langle w^{\prime}(t) \wedge w(t), w^{\prime}(t) \wedge w(t)\right\rangle=\left|\begin{array}{cc}
\left\langle w^{\prime}(t), w^{\prime}(t)\right\rangle & \left\langle w(t), w^{\prime}(t)\right\rangle \\
\left\langle w^{\prime}(t), w(t)\right\rangle & \langle w(t), w(t)\rangle
\end{array}\right|=\left\langle w^{\prime}(t), w^{\prime}(t)\right\rangle=\left\|w^{\prime}(t)\right\|^{2} .
$$

Logo,

$$
\left\|X_{t}(t, u) \wedge X_{u}(t, u)\right\|^{2}=\lambda^{2}(t)\left\|w^{\prime}(t)\right\|^{2}+u^{2}\left\|w^{\prime}(t)\right\|^{2}=\left(\lambda^{2}(t)+u^{2}\right)\left\|w^{\prime}(t)\right\|^{2}
$$

Sabemos que os pontos singulares da superfície $X$, são os pontos $\left(t_{0}, u_{0}\right) \in I \times J$ tais que $X_{t}\left(t_{0}, u_{0}\right) \wedge X_{u}\left(t_{0}, u_{0}\right)=\overrightarrow{0}$, ou seja, $\left\|X_{t}\left(t_{0}, u_{0}\right) \wedge X_{u}\left(t_{0}, u_{0}\right)\right\|=0$. Assim,

$$
0=\left(\lambda^{2}\left(t_{0}\right)+u^{2}\right) \underbrace{\left\|w^{\prime}\left(t_{0}\right)\right\|^{2}}_{\neq 0} \Longleftrightarrow \lambda^{2}\left(t_{0}\right)+u^{2}=0 \Longleftrightarrow u=\lambda\left(t_{0}\right)=0,
$$

isto é, os eventuais pontos singulares de $X$ situam-se ao longo da curva de estricção $(u=0)$, isto é, são pontos da forma $\left(t_{0}, 0\right)$ e eles ocorrem se, e somente se, $\lambda\left(t_{0}\right)=0$.

Tomando o produto interno de (6) com $w^{\prime}$, obtemos

$$
\lambda(t)=\frac{\left\langle\beta^{\prime}(t) \wedge w(t), w^{\prime}(t)\right\rangle}{\left\|w^{\prime}(t)\right\|^{2}}, t \in I
$$

Vimos que a curva de estricção independe da escolha da diretriz, segue então que, o mesmo vale para a função $\lambda$.

Vamos agora, calcular a curvatura Gaussiana da superfície regrada (4) em seus pontos regulares. Temos que: 


$$
\begin{gathered}
N(t, u)=\frac{X_{t}(t, u) \wedge X_{u}(t, u)}{\left\|X_{t}(t, u) \wedge X_{u}(t, u)\right\|}=\frac{\lambda w^{\prime}(t)+u w^{\prime}(t) \wedge w(t)}{\sqrt{\lambda^{2}(t)+u^{2}}\left\|w^{\prime}(t)\right\|}, \\
g(t, u)=\left\langle X_{u u}(t, u), N(t, u)\right\rangle=\langle\overrightarrow{0}, N(t, u)\rangle=0,(\operatorname{logo}, \text { não precisamos calcular o coeficiente } e \text { da } \\
2^{a} \text { Forma Fundamental) } \\
f(t, u)=\left\langle X_{t u}(t, u), N(t, u)\right\rangle=\frac{\lambda(t)\left\|w^{\prime}(t)\right\|}{\sqrt{\lambda^{2}(t)+u^{2}}} \Longrightarrow f^{2}(t, u)=\frac{\lambda^{2}(t)\left\|w^{\prime}(t)\right\|^{2}}{\lambda^{2}(t)+u^{2}}, \\
\left\|X_{t}(t, u) \wedge X_{u}(t, u)\right\|^{2}=\left(\lambda^{2}(t)+u^{2}\right)\left\|w^{\prime}(t)\right\|^{2}=E G-F^{2}(t, u) .
\end{gathered}
$$

Portanto,

$$
K(t, u)=\frac{e g-f^{2}}{E G-F^{2}}(t, u)=\frac{-\frac{\lambda^{2}(t)\left\|w^{\prime}(t)\right\|^{2}}{\lambda^{2}(t)+u^{2}}}{\left(\lambda^{2}(t)+u^{2}\right)\left\|w^{\prime}(t)\right\|^{2}}=-\frac{\lambda^{2}(t)}{\left(\lambda^{2}(t)+u^{2}\right)^{2}} \leq 0 .
$$

Para finalizar a demonstração, veja que, se a curvatura Gaussiana $K$ for nula em algum ponto $X\left(t_{0}, u_{0}\right)$, então ela será nula ao longo de toda a geratriz que contém esse ponto. De fato, se $K\left(t_{0}, u_{0}\right)=0$, então necessariamente teremos $\lambda\left(t_{0}\right)=0$. Uma vez fixado $t_{0} \in I$, obtemos a geratriz $L_{t_{0}}(u)=X\left(t_{0}, u\right)=\beta\left(t_{0}\right)+u w\left(t_{0}\right), u \in J$. Independentemente da variável $u, X\left(t_{0}, u\right)$ são pontos de $L_{t_{0}} \operatorname{com} \lambda\left(t_{0}\right)=0$, ou seja, todos os pontos dessa geratriz possuem curvatura Gaussiana nula. Em particular, $L_{t_{0}}$ cortará a curva de estricção $(u=0)$ no ponto singular $X\left(t_{0}, 0\right)$.

Definição 9 Seja $X: I \times J \longrightarrow \mathbb{R}^{3}$, definida por $X(t, u)=\beta(t)+u w(t)$, uma parametrização de superfície regrada não-cilíndrica, onde $\beta(t)$ é sua curva de estricção. A função $\lambda=\lambda(t)$, definida por

$$
\lambda(t)=\frac{\left\langle\beta^{\prime}(t) \wedge w(t), w^{\prime}(t)\right\rangle}{\left\|w^{\prime}(t)\right\|^{2}}, t \in I
$$

é chamada parâmetro de distribuição $d e X$.

\section{Interpretações geométricas}

\subsection{Interpretação geométrica da curva de estricção}

A equação

$$
K(t, u)=-\frac{\lambda^{2}(t)}{\left(\lambda^{2}(t)+u^{2}\right)^{2}}
$$

nos permite dar uma interpretação geométrica para a curva de estricção: ela é o lugar geométrico dos pontos da superfície regrada não-clilíndrica onde $|K(t, u)|$, para cada $t \in I$ fixado, assume seu valor máximo. De fato, pela proposição anterior, sabemos que os pontos de uma geratriz, exceto possivelmente o ponto central, são pontos regulares da superfície. Fixando uma geratriz $L_{t_{0}}$, temos que

$$
K\left(t_{0}, u\right)=-\frac{\lambda^{2}\left(t_{0}\right)}{\left(\lambda^{2}\left(t_{0}\right)+u^{2}\right)^{2}} \Longrightarrow\left|K\left(t_{0}, u\right)\right|=\left|-\frac{\lambda^{2}\left(t_{0}\right)}{\left(\lambda^{2}\left(t_{0}\right)+u^{2}\right)^{2}}\right|=\left(\frac{\lambda\left(t_{0}\right)}{\lambda^{2}\left(t_{0}\right)+u^{2}}\right)^{2} .
$$


Se $\lambda\left(t_{0}\right) \neq 0$, a função $\left|K\left(t_{0}, u\right)\right|$ é uma função contínua sobre a geratriz $L_{t_{0}}$. Essa função assume valor máximo, quando seu denominador é mínimo. $\mathrm{E}$, isso ocorre obrigatoriamente no ponto em que $u=0$, isto é, no ponto central $\beta\left(t_{0}\right)$ da superfície regrada. Logo, a curva de estricção de uma superfície regrada não-cilíndrica é o lugar geométrico dos pontos onde as funções $|K(t, u)|$ assumem seus valores máximos.

Considerando os pontos sobre uma geratriz $L_{t_{0}}$ que são simétricos em relação ao ponto central $\beta\left(t_{0}\right)$, isto é, os pontos $X\left(t_{0},-u\right)$ e $X\left(t_{0}, u\right), u \neq 0$, temos que:

$$
K\left(t_{0}, u\right)=-\frac{\lambda\left(t_{0}\right)}{\left(\lambda^{2}\left(t_{0}\right)+u^{2}\right)^{2}}=-\frac{\lambda\left(t_{0}\right)}{\left(\lambda^{2}\left(t_{0}\right)+(-u)^{2}\right)^{2}}=K\left(t_{0},-u\right),
$$

ou seja, a curvatura Gaussiana $K$ de uma superfície regrada não-cilíndrica, assume os mesmos valores em pontos sobre uma geratriz fixada que são simétricos em relação ao ponto central (isso justifica o adjetivo central para os pontos da curva de estricção).

\section{Exemplos:}

(1) Vimos que a curva de estricção do hiperboloide de uma folha $X(s, v)=(\cos (s)-v \operatorname{sen}(s)$, $\operatorname{sen}(s)+$ $v \cos (s), v),(s, v) \in(0-\varepsilon, 2 \pi+\varepsilon) \times \mathbb{R}, \varepsilon>0$, é a circunferência unitária $\mathbb{S}^{1}$. Considere a geratriz

$$
L_{\frac{\pi}{4}}=X\left(\frac{\pi}{4}, v\right)=\left(\frac{\sqrt{2}}{2}-v \frac{\sqrt{2}}{2}, \frac{\sqrt{2}}{2}+v \frac{\sqrt{2}}{2}, v\right),
$$

e os pontos $X\left(\frac{\pi}{4}, 1\right)=(0, \sqrt{2}, 1)$ e $X\left(\frac{\pi}{4},-1\right)=(\sqrt{2}, 0,-1)$, pertencentes à essa geratriz e simétricos em relação ao ponto central $X\left(\frac{\pi}{4}, 0\right)=\left(\frac{\sqrt{2}}{2}, \frac{\sqrt{2}}{2}, 0\right)$.

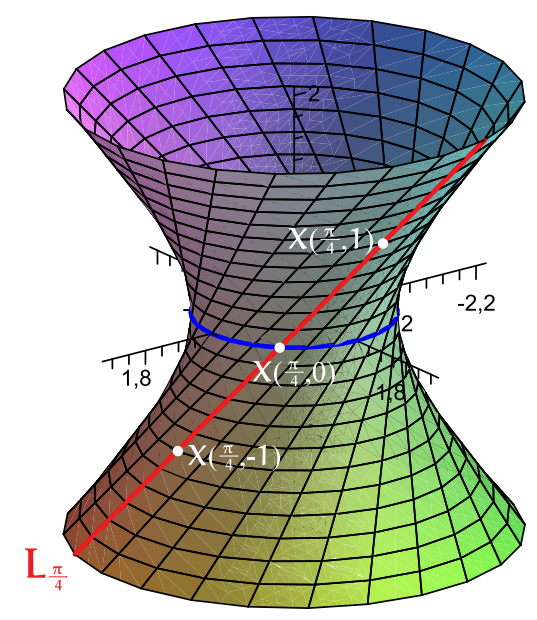

Figura 12: Pontos simétricos da geratriz $L_{\frac{\pi}{4}}$

Calculando a função parâmetro de distribuição de $X$, obtemos:

$$
\lambda(s)=\frac{\left\langle\beta^{\prime}(s) \wedge w(s), w^{\prime}(s)\right\rangle}{\left\|w^{\prime}(s)\right\|^{2}}=\frac{-1}{1}=-1,
$$


para todo $s \in(0-\varepsilon, 2 \pi+\varepsilon), \varepsilon>0$. Vimos também que as singularidades de uma superfície regrada não-cilíndrica estão sobre a curva de estricção e elas ocorrem se, e somente se, $\lambda(s)=0$. Como $\lambda \equiv 1$, concluímos que o hiperboloide de uma folha não possui pontos singulares. A curvatura Gaussiana em pontos regulares é dada por:

$$
K(s, v)=-\frac{\lambda^{2}(s)}{\left(\lambda^{2}(s)+v^{2}\right)^{2}}=\frac{-1}{\left(1+v^{2}\right)^{2}} .
$$

Assim, nos pontos considerados acima, temos:

$$
K\left(\frac{\pi}{4}, 1\right)=-\frac{1}{4}=K\left(\frac{\pi}{4},-1\right) .
$$

(2) Calculando a função parâmetro de distribuição do guarda-chuva de Whitney, obtemos:

$$
\lambda(t)=\frac{\left\langle\beta^{\prime}(t) \wedge w(t), w^{\prime}(t)\right\rangle}{\left\|w^{\prime}(t)\right\|^{2}}=\frac{2 t}{\left(1+t^{2}\right)\left\|w^{\prime}(t)\right\|^{2}} .
$$

Daí, $\lambda(t)=0$ se, e somente se, $t=0$, ou seja, o único ponto singular do guarda-chuva de Whitney está em $(0,0,0)$.

\subsection{Interpretação geométrica para a função parâmetro de distribuição}

Vamos mostrar que, se $X$ é uma superfície regrada não-cilíndrica regular e $\theta$ é o ângulo que o vetor normal em um ponto de uma geratriz faz com o vetor normal no ponto central desta geratriz, então a $\operatorname{tg}(\theta)$ é proporcional à distância entre estes dois pontos, e o coeficiente de proporcionalidade é o inverso do parâmetro de distribuição $\lambda$.

De fato, temos que o vetor normal unitário à superfície regrada não-cilíndrica $X(t, u)=\beta(t)+$ $u w(t)$, sendo $\beta$ a curva de estricção, $\|w(t)\| \equiv 1$ e $w^{\prime}(t) \neq \overrightarrow{0}, \forall t \in I$, é dado por:

$$
N(t, u)=\frac{X_{t}(t, u) \wedge X_{u}(t, u)}{\left\|X_{t}(t, u) \wedge X_{u}(t, u)\right\|}=\frac{\lambda(t) w^{\prime}(t)+u w^{\prime}(t) \wedge w(t)}{\sqrt{\lambda^{2}(t)+u^{2}}\left\|w^{\prime}(t)\right\|} .
$$

Como estamos supondo $X$ regular, em cima da curva de estricção $(u=0)$ devemos ter $\lambda(t) \neq 0$. Logo, para os pontos centrais da superfície, temos

$$
N(t, 0)=\frac{w^{\prime}(t) \lambda(t)}{|\lambda(t)| \| w^{\prime}(t)||}
$$

Se $\theta$ é o ângulo formado por $N(t, u)$ e $N(t, 0)$, temos que:

$$
\begin{aligned}
\cos (\theta) & =\frac{\langle N(t, u), N(t, 0)\rangle}{\|N(t, u)\|\|N(t, 0)\|}=\langle N(t, u), N(t, 0)\rangle=\frac{|\lambda(t)|}{\sqrt{\lambda^{2}(t)+u^{2}}} \\
\operatorname{sen}(\theta) & =\frac{\|N(t, u) \wedge N(t, 0)\|}{\|N(t, u)\|\|N(t, 0)\|}=\frac{u}{\sqrt{\lambda^{2}(t)+u^{2}}},
\end{aligned}
$$

uma vez que $(\vec{u} \wedge \vec{v}) \wedge \vec{w}=\langle\vec{u}, \vec{w}\rangle \vec{v}-\langle\vec{v}, \vec{w}\rangle \vec{u}$. Logo, 


$$
\operatorname{tg}(\theta)=\frac{u}{|\lambda(t)|}
$$

como queríamos.

\section{Superfícies regradas desenvolvíveis}

Consideremos uma superfície regrada arbitrária (não necessariamente não-cilíndrica)

$$
X(t, v)=\alpha(t)+v w(t)
$$

gerada pela família $\{\alpha(t), w(t)\}$, com $\|w(t)\|=1$. Dizemos que essa superfície é desenvolvível quando $\left(w(t), w^{\prime}(t), \alpha^{\prime}(t)\right)=0$, para todo $t$, onde $\left(w(t), w^{\prime}(t), \alpha^{\prime}(t)\right)$ denota o produto misto dos vetores $w(t), w^{\prime}(t)$ e $\alpha^{\prime}(t)$, isto é, $\left(w(t), w^{\prime}(t), \alpha^{\prime}(t)\right)=\left\langle w(t) \wedge w^{\prime}(t), \alpha^{\prime}(t)\right\rangle$.

O próximo resultado nos dá uma interpretação geométrica para $\left(w, w^{\prime}, \alpha^{\prime}\right) \equiv 0$.

Proposição 10 Se uma superfície regrada é desenvolvível, então sua curvatura Gaussiana é identicamente nula em pontos regulares.

Demonstração: Seja $X(t, v)=\alpha(t)+v w(t)$ uma superfície regrada desenvolvível, ou seja, $\left(w, w^{\prime}, \alpha^{\prime}\right) \equiv$ 0 . Nos pontos regulares de $X$, temos que:

$$
\begin{aligned}
X_{t}(t, v) & =\alpha^{\prime}(t)+v w^{\prime}(t) \\
X_{v}(t, v) & =w(t) \\
X_{t}(t, v) \wedge X_{v}(t, v) & =\alpha^{\prime}(t) \wedge w(t)+v w^{\prime}(t) \wedge w(t) \\
X_{t t}(t, v) & =\alpha^{\prime \prime}(t)+v w^{\prime \prime}(t) \\
X_{v v}(t, v) & =\overrightarrow{0} \\
X_{t v}(t, v) & =w^{\prime}(t) \\
N(t, v) & =\frac{X_{t}(t, v) \wedge X_{v}(t, v)}{\left\|X_{t}(t, v) \wedge X_{v}(t, v)\right\|} .
\end{aligned}
$$

Logo,

$$
\begin{aligned}
g(t, v) & =\left\langle X_{v v}(t, v), N(t, v)\right\rangle=\langle\overrightarrow{0}, N(t, v)\rangle=0, \\
f(t, v) & =\left\langle X_{t v}(t, v), N(t, v)\right\rangle \\
& =\left\langle w^{\prime}(t), \frac{\alpha^{\prime}(t) \wedge w(t)+v w^{\prime}(t) \wedge w(t)}{\left\|X_{t}(t, v) \wedge X_{v}(t, v)\right\|}\right\rangle
\end{aligned}
$$




$$
\begin{aligned}
& =\frac{1}{\left\|X_{t}(t, v) \wedge X_{v}(t, v)\right\|}\left\langle w^{\prime}(t), \alpha^{\prime}(t) \wedge w(t)\right\rangle \\
& =\frac{1}{\left\|X_{t}(t, v) \wedge X_{v}(t, v)\right\|}\left\langle\alpha^{\prime}(t) \wedge w(t), w^{\prime}(t)\right\rangle \\
& =\frac{1}{\left\|X_{t}(t, v) \wedge X_{v}(t, v)\right\|}\left(\alpha^{\prime}(t), w(t), w^{\prime}(t)\right) \\
& =\frac{1}{\left\|X_{t}(t, v) \wedge X_{v}(t, v)\right\|} \underbrace{\left(w(t), w^{\prime}(t), \alpha^{\prime}(t)\right)}_{=0}=0 .
\end{aligned}
$$

Portanto, temos que:

$$
K(t, v)=\frac{e g-f^{2}}{E G-F^{2}}(t, v)=0,
$$

como queríamos.

Os exemplos mais simples de superfícies regradas desenvolvíveis são o plano, os cilindros circulares e os cones. Outra propriedade interessante das superfícies regradas desenvolvíveis é dada na proposição a seguir:

Proposição 11 Se $X(t, v)=\alpha(t)+v w(t)$ é uma superfície regrada desenvolvível, então o plano tangente a essa superfície é constante ao longo dos pontos regulares de uma geratriz fixada.

Demonstração: Vamos mostrar que, em pontos regulares, o vetor normal $N(t, v)$ do plano tangente à superfície desenvolvível dada não depende de $v$. Assim, o plano tangente será constante ao longo (dos pontos regulares) de uma geratriz fixada. Em pontos regulares, temos que:

$$
\begin{aligned}
X_{t}(t, v) & =\alpha^{\prime}(t)+v w^{\prime}(t) \\
X_{v}(t, v) & =w(t) \\
X_{t}(t, v) \wedge X_{v}(t, v) & =\alpha^{\prime}(t) \wedge w^{\prime}(t)+v w^{\prime}(t) \wedge w(t) \\
N(t, v) & =\frac{X_{t}(t, v) \wedge X_{v}(t, v)}{\left\|X_{t}(t, v) \wedge X_{v}(t, v)\right\|}=\frac{\alpha^{\prime}(t) \wedge w^{\prime}(t)+v w^{\prime}(t) \wedge w(t)}{\left\|X_{t}(t, v) \wedge X_{v}(t, v)\right\|} \\
N_{v}(t, v) & =\frac{1}{\left\|X_{t}(t, v) \wedge X_{v}(t, v)\right\|} w^{\prime}(t) \wedge w(t) .
\end{aligned}
$$

Agora, veja que:

$$
\begin{aligned}
\left\langle N_{v}(t, v), X_{t}(t, v)\right\rangle & =\frac{1}{\left\|X_{t}(t, v) \wedge X_{v}(t, v)\right\|}\left[\left\langle w^{\prime}(t) \wedge w(t), \alpha^{\prime}(t)\right\rangle+v\left\langle w^{\prime}(t) \wedge w(t), w^{\prime}(t)\right\rangle\right] \\
& =0
\end{aligned}
$$

pois, $\left\langle w^{\prime}(t) \wedge w(t), \alpha^{\prime}(t)\right\rangle=\left\langle w(t) \wedge w^{\prime}(t), \alpha^{\prime}(t)\right\rangle=0$ (definição de superfície desenvolvível) e $\left\langle w^{\prime}(t) \wedge w(t), w^{\prime}(t)\right\rangle=0$ (propriedade de produto vetorial). E, analogamente, 


$$
\left\langle N_{v}(t, v), X_{v}(t, v)\right\rangle=\frac{1}{\left\|X_{t}(t, v) \wedge X_{v}(t, v)\right\|}\left\langle w^{\prime}(t) \wedge w(t), \alpha^{\prime}(t)\right\rangle=0
$$

Assim, $N_{v}(t, v) \perp X_{t}(t, v)$ e $N_{v}(t, v) \perp X_{v}(t, v)$. Daí, segue que $N_{v}(t, v)$ é paralelo a $X_{t}(t, v) \wedge X_{v}(t, v)$, ou seja,

$$
N_{v}(t, v) / / N(t, v) .
$$

Por outro lado, como $\|N(t, v)\|=1$, temos que $\|N(t, v)\|^{2}=\langle N(t, v), N(t, v)\rangle=1$. Derivando essa expressão em relação a $v$, obtemos:

$$
2\left\langle N_{v}(t, v), N(t, v)\right\rangle=0 \Longrightarrow\left\langle N_{v}(t, v), N(t, v)\right\rangle=0 .
$$

Logo, de (11) e (12), temos que $N_{v} \equiv 0$. Portanto, $N(t, v)$ não depende de $v$ e, consequentemente, fixada uma geratriz $L_{t_{0}}$, temos que $N\left(t_{0}, v\right)$ é constante ao longo de $L_{t_{0}} \mathrm{e}$, assim, o plano tangente à superfície desenvolvível é constante ao longo dessa geratriz.

Antes de darmos o próximo exemplo, vamos precisar da seguinte definição:

Definição 12 Seja $p$ um ponto em uma superfície regular $S$. Uma direção assintótica de $S$ em $p$ é uma direção de $T_{p} S$ para a qual a curvatura normal é zero. Uma curva assintótica de $S$ é uma curva conexa e regular $C \subset S$ tal que para cada $p \in C$, a reta tangente a $C$ em $p$ é uma direção assintótica.

Exemplo: Seja $S$ uma superfície regular e $\alpha(s)$ uma curva em $S$ parametrizada pelo comprimento de arco. Suponhamos que $\alpha$ não é tangente a uma direção assintótica, isto é, $\alpha^{\prime}(s)$ não é uma direção assintótica e, portanto,

$$
k_{n}(s)=I I\left(\alpha^{\prime}(s)\right)=-\left\langle d N\left(\alpha^{\prime}(s)\right), \alpha^{\prime}(s)\right\rangle \neq 0 .
$$

Assim, $d N\left(\alpha^{\prime}(s)\right)=N^{\prime}(s) \neq \overrightarrow{0}$. A superfície regrada parametrizada por

$$
X(s, v)=\alpha(s)+v \frac{N(s) \wedge N^{\prime}(s)}{\left\|N^{\prime}(s)\right\|},(s, v) \in I \times J,
$$

onde $N(s)$ é o vetor normal unitário de $S$ restrito à curva $\alpha(s)$, é chamada envoltória da família de planos tangentes à $S$ ao longo de $\alpha(s)$.

Vamos mostrar que $X$ é desenvolvível, que é regular em uma vizinhança de $v=0$ e que é tangente à $S$ ao longo de $v=0$. Além disso, daremos uma interpretação geométrica dessa superfície regrada.

Mostremos que a superfície dada em (13) é uma superfície regrada desenvolvível. De fato, temos que $X$ é gerada pela família $\alpha(s)$ e $w(s)=\frac{N(s) \wedge N^{\prime}(s)}{\left\|N^{\prime}(s)\right\|}$. Daí, para todo $s$ em $I$, tem-se que: 


$$
\begin{aligned}
\left(w(s), w^{\prime}(s), \alpha^{\prime}(s)\right) & =\left\langle w(s) \wedge w^{\prime}(s), \alpha^{\prime}(s)\right\rangle \\
& =\left\langle\frac{N(s) \wedge N^{\prime}(s)}{\left\|N^{\prime}(s)\right\|} \wedge\left(\frac{N(s) \wedge N^{\prime}(s)}{\left\|N^{\prime}(s)\right\|}\right)^{\prime}, \alpha^{\prime}(s)\right\rangle \\
& =\frac{1}{\left\|N^{\prime}(s)\right\|^{2}}\left\langle\left\langle N(s) \wedge N^{\prime}(s), N^{\prime \prime}(s)\right\rangle N(s), \alpha^{\prime}(s)\right\rangle=0,
\end{aligned}
$$

uma vez que $N(s)$ é unitário e $(\vec{u} \wedge \vec{v}) \wedge \vec{w}=\langle\vec{u}, \vec{w}\rangle \vec{v}-\langle\vec{v}, \vec{w}\rangle \vec{u}$. Portanto, $X$ é uma superfície regrada desenvolvível.

Provaremos agora que $X$ é regular em uma vizinhança de $v=0$ e é tangente à $S$ ao longo de $\alpha(s)$. De fato, em $v=0$, temos

$$
\begin{aligned}
X_{s}(s, 0) \wedge X_{v}(s, 0) & =\alpha^{\prime}(s) \wedge\left(\frac{N(s) \wedge N^{\prime}(s)}{\left\|N^{\prime}(s)\right\|}\right) \\
& =\left\langle N^{\prime}(s), \alpha^{\prime}(s)\right\rangle \frac{N(s)}{\left\|N^{\prime}(s)\right\|}=-k_{n}(s) \frac{N(s)}{\left\|N^{\prime}(s)\right\|}
\end{aligned}
$$

onde $k_{n}(s)$ é a curvatura normal de $\alpha(s)$. Portanto, como $k_{n}(s)$ é não nula, temos que $X$ é regular numa vizinhança de $v=0$, e como

$$
X_{s}(s, 0) \wedge X_{v}(s, 0)=-k_{n}(s) \frac{N(s)}{\left\|N^{\prime}(s)\right\|}, \forall s \in I,
$$

segue que o vetor normal unitário de $X$ em $X(s, 0)$ é paralelo a $N(s)$, ou seja, $X$ é tangente à $S$ ao longo de $v=0$, como queríamos.

Por fim, vamos obter uma interpretação geométrica para $X$. Para isso, considere a família $\left\{T_{\alpha(s)} S\right\}$ de planos tangentes à superfície $S$ ao longo da curva $\alpha(s)$. Se $\Delta s$ é pequeno, dois planos $T_{\alpha(s)} S$ e $T_{\alpha(s)+\Delta s} S$ da família intersectam-se ao longo de uma reta paralela ao vetor

$$
\frac{N(s) \wedge N(s+\Delta s)}{\Delta s}
$$

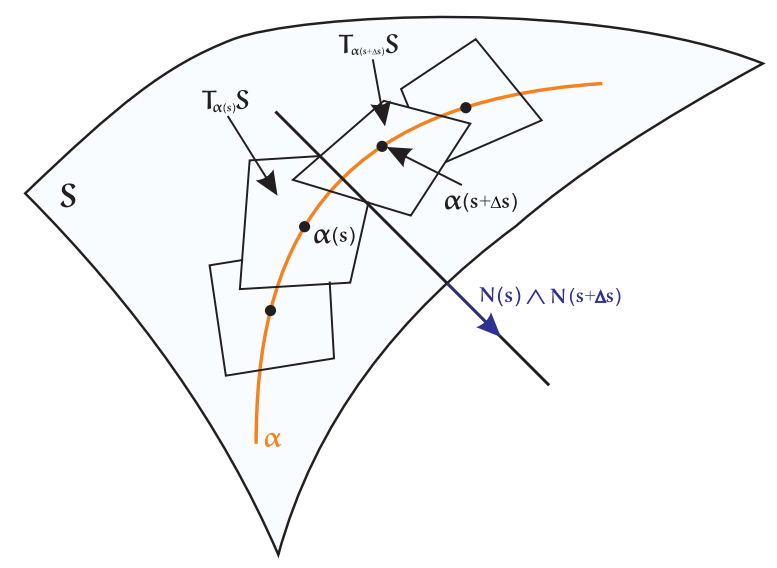

Figura 13: Intersecção de planos tangentes ao longo de $\alpha$ 
Se fizermos $\Delta s$ tender a zero, esta reta se aproxima de uma posição limite paralela ao vetor

$$
\lim _{\Delta s \rightarrow s} \frac{N(s) \wedge N(s+\Delta s)}{\Delta s}=\lim _{\Delta s \rightarrow s} N(s) \wedge \frac{(N(s+\Delta s)-N(s))}{\Delta s}=N(s) \wedge N^{\prime}(s) .
$$

Intuitivamente, isto significa que as geratrizes de $X$ são as posições limites da intersecção de planos vizinhos da família $\left\{T_{\alpha(s)} S\right\}$. Isto justifica também o nome que atribuímos à superfície regrada desenvolvível $X$.

Como exemplos, temos que, se $\alpha(s)$ é uma parametrização de um paralelo da esfera $\mathbb{S}^{2}$, então a envoltória de planos tangentes de $\mathbb{S}^{2}$ ao longo de $\alpha(s)$ é um cilindro, se o paralelo for o equador, ou um cone, se o paralelo não for um equador.
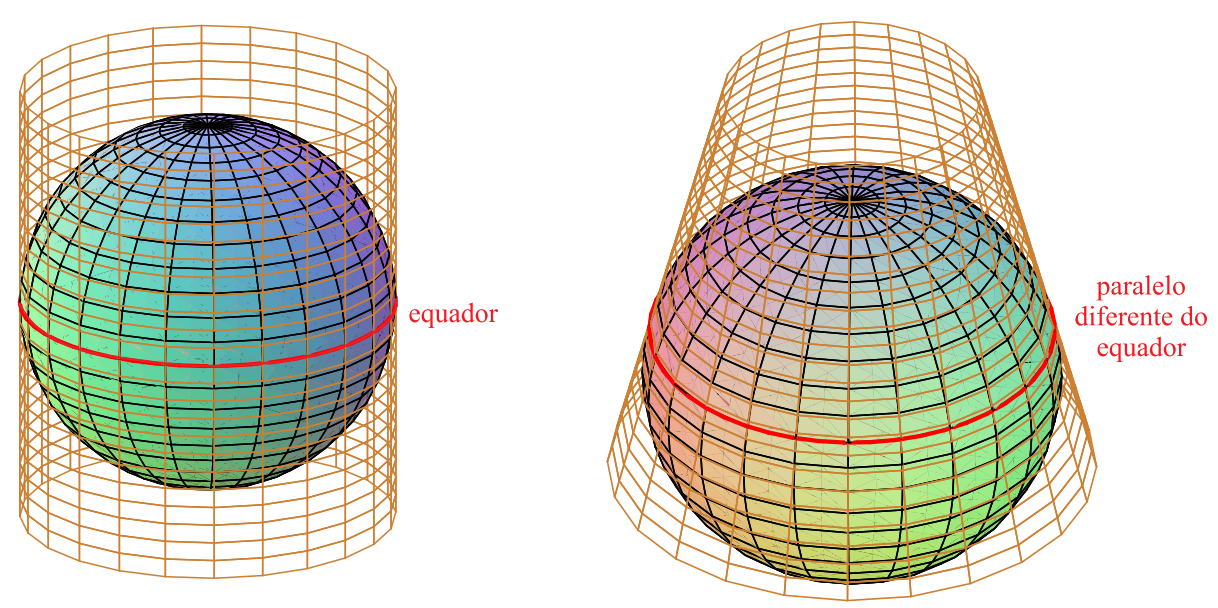

Figura 14: Envoltória de planos tangentes a uma esfera ao longo de um paralelo

\subsection{Classificação Parcial das Superfícies Desenvolvíveis}

Vamos provar um Teorema de Classificação Parcial para as superfícies regradas desenvolvíveis, no qual fazemos o estudo de dois casos distintos (sem exaurir as possibilidades).

Teorema 13 Seja $X(t, v)=\alpha(t)+v w(t),(t, v) \in I \times J$, uma superfície regrada desenvolvível gerada pela família $\{\alpha(t), w(t)\}$, com $\|w(t)\|=1, \forall t \in I$.

(a) Se $w^{\prime} \equiv \overrightarrow{0}$, então a superfície regrada é um cilindro generalizado.

(b) Se $w^{\prime}(t)$ nunca se anula, isto é, se $X$ é não-cilíndrica, podemos considerar sua curva de estricção $\beta$ e temos:

(b.1) $\operatorname{Se} \beta^{\prime}(t) \neq \overrightarrow{0}$, para todo $t \in I$, então $X$ é a superfície tangente de sua curva de estricção.

(b.2) Se $\beta^{\prime}(t)=\overrightarrow{0}$, para todo $t \in I$, então $X$ é um cone generalizado.

Demonstração: $(a)$ Como $w^{\prime} \equiv \overrightarrow{0}$, então $w(t)$ é vetor constante para todo $t$ em $I$. Assim, $X$ é gerada pela família $\{\alpha(t), w(t)\}$, com $w(t)$ constante, ou seja, $X$ é um cilindro generalizado. 
(b.1) Como $X$ é desenvolvível, temos que $\left(\beta^{\prime}(t), w(t), w^{\prime}(t)\right)=0, \forall t \in I$ (uma vez que o parâmetro de distribuição de $X$ independe da escolha da diretriz) e, por hipótese, temos que $\beta^{\prime}(t) \neq \overrightarrow{0}$, para todo $t \in I$. Assim, o produto misto de $\beta^{\prime}(t), w(t)$ e $w^{\prime}(t)$ é identicamente nulo, sem que nenhum dos vetores envolvidos seja o vetor nulo. Logo, pelo menos um dos vetores do produto misto deve ser proporcional a outro envolvido. Como $\left\langle\beta^{\prime}(t), w^{\prime}(t)\right\rangle=0$, segue que $\beta^{\prime}(t)$ e $w^{\prime}(t)$ não são proporcionais, bem como $w(t)$ e $w^{\prime}(t)$ (pois, $\left\langle w(t), w^{\prime}(t)\right\rangle=0$ ). Portanto, temos que $w(t)$ é paralelo a $\beta^{\prime}(t)$, ou seja, a superfície regrada é a superfície tangente da curva de estricção $\beta$, visto que podemos considerar uma reparametrização para $X$, tendo como diretriz a sua curva de estricção.

Observe que, neste caso, o lugar geométrico dos pontos singulares da superfície regrada é exatamente sua curva de estricção. De fato, vimos que a função parâmetro de distribuição de $X$ é dada por

$$
\lambda(t)=\frac{\left(\beta^{\prime}(t), w(t), w^{\prime}(t)\right)}{\left\|w^{\prime}(t)\right\|^{2}}
$$

Como $X$ é desenvolvível, temos que $\left(\beta^{\prime}(t), w(t), w^{\prime}(t)\right)=0, \forall t \in I$. Logo,

$$
\lambda(t)=\frac{\left(\beta^{\prime}(t), w(t), w^{\prime}(t)\right)}{\left\|w^{\prime}(t)\right\|^{2}}=0, \text { para todo } t \in I .
$$

Portanto, a curva de estricção é o lugar geométrico dos pontos singulares da superfície desenvolvível, como queríamos.

(b.2) Se $\beta^{\prime}(t)=\overrightarrow{0}$, para todo $t \in I$, então a curva de estricção é um ponto e assim, $X(t, u)=$ $p+u w(t)$, isto é, $X$ é um cone generalizado.

\section{Observações:}

(i) Novamente, ressaltamos que o Teorema acima não exauri, de modo algum, todas as possiblidades de classificação para as superfícies regradas desenvolvíveis. Se os zeros das funções $\beta$ e $w$ apresentarem pontos de acumulação, então as superfícies regradas desenvolvíveis dadas por esta família, podem ser mais complicadas. Em qualquer um dos casos, longe dos pontos de acumulação dos zeros das funções envolvidas, uma superfície regrada desenvolvível é uma união de pedaços de cilindros, cones e superfícies tangentes. Uma prova deste fato pode ser encontrada em [1], página 185. Toda superfície regrada desenvolvível possui uma parametrização para suas geratrizes dada por $(u, v) \longmapsto X(u, v)$, a qual pode ser subdividida em $n$ intervalos suficientemente pequenos de modo que cada parte da superfície correspondente a cada subintervalo é um subconjunto de uma das formas: um plano, um cilindro, um cone ou uma superfície tangente.

(ii) O estudo de superfícies regradas é um assunto clássico em Geometria Diferencial [2, 3, 4]. $\mathrm{O}$ interesse pelo tema tem reaparecido atualmente em diferentes áreas, como Geometria Diferencial Projetiva [5], Computação Gráfica [6] e Desenho Industrial [7]. 
(iii) Vimos que, em geral, as superfícies regradas possuem singularidades. Existem vários resultados interessantes envolvendo as mesmas. Um estudo desse tipo é mais avançado e está além dos propósitos desse trabalho. No entanto, resultados dessa natureza podem ser encontrados, por exemplo, em [8]. Dentre os vários resultados, o autor, mostra que embora o conjunto das superfícies regradas seja um subconjunto pequeno no espaço $C^{\infty}\left(\mathbb{R}^{2}, \mathbb{R}^{3}\right)$ de todas as superfícies parametrizadas, as singularidades genéricas que ocorrem no conjunto das superfícies regradas são as mesmas que ocorrem no conjunto todo e que a única singularidade genérica que ocorre no conjunto dos germes das superfícies é o guarda-chuva de Whitney. Além disso, o autor faz uma comparação do comportamento genérico das singularidades das superfícies regradas com as singularidades genéricas da subclasse das superfícies desenvolvíveis.

\section{Referências}

[1] KREYSZIG, E. Introduction to differential geometry and Riemannian geometry. Toronto: University of Toronto Press, 1968. Reprint by Dover Publication, New York, 1991.

[2] CARMO, M. P. Geometria diferencial de curvas e superfícies. Rio de Janeiro: SBM, 2005.

[3] GRAY, A.; ABBENA, E.; SALAMON, S. Modern differential geometry of curves and surfaces with Mathematica. 3. ed. Boca Raton: Chapman \& Hall CRC, 2006.

[4] TENENBLAT, K. Introdução à geometria diferencial. Brasília: Editora da UNB, 1988.

[5] SASAKI, T. Projective differential geometry and linear differential equations. Rokko Lectures in Mathematics, Kobe, v. 5, p. 1-114, 1999.

[6] HOSCHEK, J.; POTTMAN, H. Interpolation and approximation with developable B-spline surfaces. In: INTERNATIONAL CONFERENCE ON MATHEMATICAL METHODS FOR CURVES AND SURFACES, 1995, Nashville. Proceedings...Vanderbilt University, 1995. p. 255-264.

[7] SCHNEIDER, M. Interpolation with developable strip-surfaces consisting of cylinders and cones. In: INTERNATIONAL CONFERENCE ON MATHEMATICAL METHODS FOR CURVES AND SURFACES, 2., 1998, Nashville. Proceedings... Nashville: Vanderbilt University, 1998, p. 437-444.

[8] MARTINS, R. Singularidades das superfícies regradas em R $\mathbf{R}^{3}$. 2004. 49 f. Dissertação (Mestrado em Matemática)- Instituto de Ciências Matemáticas e de Computação, Universidade de São Paulo, São Carlos, 2004. 